\title{
Rise and Recent Hasten In Image Processing and Healthcare \\ A Swarnambiga*
}

Department of Electronics and Communication Engineering, SACS MAVMM Engineering College, Madurai, India

\begin{abstract}
To increase collaboration and to consistently deliver higher value, the health care industry needs to be streamlined and evidence based initiative are needed to increase. A number of evidence based initiatives are needed to eliminate unneeded lab test and to increase patient comfort. Human vision limited to volumes and also cannot perceive variations in volume of any images, but now it is possible to count these volumes accurately with statistically proven results. Nowadays, the use of image processing is an emerging biomedical tool contributing for the betterment of healthcare sector.
\end{abstract}

\section{Expert Review}

It is believed and proved that images bear the greatest density of natural information on all ways and opens the door for human communication. Even biomedical images do not make any exception to this assertion, when dealing with morphological information. In recent years, the key components of clinical activity are image guided therapy (IGT) and image guided surgery (IGS), and here localization, targeting, monitoring and control are the main issues. This domain medical image processing itself is interesting and it is interdisciplinary and multidisciplinary nature. Biomedical image processing is executed in multiple dimensions (2D, 3D).The packages such as Visualization Toolkit (VTK), ITK, FSL, Statistical Parametric Mapping (SPM) for generic purpose, GIMIAS, Niftyseg for automatic segmentation, 3D slicer, ITK-snap, MITK for visualization and analysis, Niftyreg, Elastix ANTS for registration, NiftyRec for reconstruction, NiftySim for simulation, Camino for diffusion and DTI-TK for spatial normalization and atlas construction helps in effective diagnosis.

Based on the diagnosis, image processing can be used to determine and analyze for instance, the diameter, volume and vascular of a tumor or organ, flow parameters of blood or other fluids and microscopic changes that have yet to raise any otherwise discernible flags. The engineering field is contributing to a remarkable synergy of efforts to achieve dynamic, quantitative (structural (or) functional) imaging of the body using minimally invasive, non-invasive or even virtual methods. By means of molecular imaging and laboratory, imaging techniques, the structural and functional relationships between the cells, tissues, organs and organ systems of the body are being advanced. Hence, we can fully accept the beneficiaries from new knowledge about health care and from new and efficient methods of diagnosis therapy and prevention.

The revolution in personal medical technology will be creating a next generation of highly useful devices and sensors which sends data to the care providers. This resolution will tell doctors what they really need to know about their patients when they need to know it. These fields of research will prove transformative in the development of new therapies and exciting frontiers in medicine. Researchers widely lack access to expertise and resources especially for medical data due to the lack of ethical clearance, which can allow them to rethink and reimagine their work, or to navigate common product development changes.

Ahead in digital health, lead to more premise, effective and even preventive treatment of human diseases. The top, cutting-edge trends for 2014 in health and science are cancer immunotherapy, Genome editing, Research in Micro biome, patient centered medical home, cell therapy, Early translation and Health Care Value.
Enormous technological changes in medicine and healthcare are heading our way. To get an overview of the trends in healthcare technology, Telemedicine and remote car by autonomous robots, such as Robot's RP-VITA, Surgical and humanoid robots, Genomics and truly personalized medicine, Body sensors, Medical recorders and portable diagnosis, 3D printing revolution, powered exoskeletons and prosthetics were leading.

There are nearly 10 technologies found to be raised and they are Digitizing the medical data, Humanizing clinical encounters with Google glass, Electronic medical assistant: structured data in a touchscreen, Medical mobile applications, Enabling price transparency for point-of-service collections, A single app with a suite of medical references, Converting a iPhone into Digital Otoscope, Activating patients by integrating devices, Plug-and-play electronic health record for e-prescribing, Taking the physical examinations on the road are in rise.

\section{Conclusion}

In this regard, a key development in health care sector has been the creation of high sped networks that enable transfer, sharing and archiving of images and related data. Whenever, a suitable clinical application is required, software based solutions have fewer constraints and have the potential to combine data from desired modalities. In the recent year's health care sector and imaging solutions have gone beyond effective diagnosis to provide case based reasoning, treatment planning, response evaluation, guiding intervention, monitoring response to the treatment and surgical planning. Due to the evolution, recent areas are found to be grown and they are, pre-surgical evaluation and planning, surgical/ treatment guidance, treatment response, and evidence based medicine. New solutions will continue through research for, new algorithms continue to be vital to finding solutions for certain clinical problems. A system to help and automate the overall workflow with significant reduction in per case time for radiologists is still needed.

${ }^{*}$ Corresponding author: A Swarnambiga, Assistant Professor, Department of Electronics and Communication Engineering, SACS MAVMM Engineering College, Madurai, India, Tel: 0452-255510; E-mail: aswarnambiga@gmail.com

Received September 14, 2015; Accepted September 16, 2015; Published September 22, 2015

Citation: Swarnambiga A (2015) Rise and Recent Hasten In Image Processing and Healthcare. Reality. J Health Med Informat 6: e137. doi:10.4172/2157-7420.1000e137

Copyright: (c) 2015 Swarnambiga A. This is an open-access article distributed under the terms of the Creative Commons Attribution License, which permits unrestricted use, distribution, and reproduction in any medium, provided the original author and source are credited. 